\title{
The Use of Rifampicin in the Treatment of Leprosy
}

\author{
E. J. SAERENS \\ University of A ntwerp, Dept. of Int. Medicine, \\ Universiteitsplein 1, Wilrijk, Belgium
}

\begin{abstract}
Rifampicin is the most active drug in the experimental mouse footpad infection with Myco. leprae and in the treatment of human lepromatous leprosy as judged by the rapid decline in Morphological Index.

Its definite place in the overall treatment of human leprosy needs however to be further ascertained through controlled clinical trials exploring different therapeutics, also intermittent and therefore less expensive regimens associated with careful patient monitoring for side-effects.
\end{abstract}

As an introduction to our discussion planned for tomorrow, I would like to raise four questions that have received only incomplete answers to my knowledge, and that I hope may provoke suggestions and lead to further investigations.

The first introductory question is: how active is rifampicin as an agent against Myco. leprae under experimental conditions? This question may seem superfluous after all the work that has been done and published by many of those here present. We do know that rifampicin administration decreases very rapidly the number of viable bacilli or is followed by a delay in growth in the mouse footpad model, and also that its minimal inhibitory concentration is somewhere in the range of 0.06 to $0.12 \mathrm{mcg} / \mathrm{ml}$ and that much higher serum levels can be reached by oral administration, as well in mice as in men.

We also know from comparative investigations that the effect of a single dose of rifampicin is of the same magnitude as that obtained by DDS given continuously for 2 months. An equally favourable impression is gained from the experimental model developed in this institute and using Myco. marinum.

Existing models with Myco. leprae have, however, their limitations: for instance the bacteriological yield does not seem to allow any deduction as far as frequency of selection of resistant mutants is concerned.

The armadillo and to a lesser extent some rat strains might provide a more suitable model in this regard but to my knowledge this question remains open. This could nevertheless be of considerable importance in the extrapolation of experimental therapeutic animal data to human therapy, (for example when we think in terms of relapse in tuberculosis due to selection of resistant mutants under monotherapy).

I am aware of the fact that if little attention has been given to the subject, it is not mainly because of lack of concern but because of lack of an appropriate model and suitable standards of comparison. 
I would now like to turn to the second question: how active is rifampicin in the therapy of human leprosy? A few hundreds of patients either treated unsuccessfully with other drugs or previously untreated have been subjected to rifampicin administration and monitored mainly in open trials or more rarely in controlled trials. Dosage has varied from 150 to $1.200 \mathrm{mg}$ per administration and the total duration has ranged from a few months to more than 2 years. Treatment regimens have consisted of continuous daily or varied intermittent schemes.

Evaluation of therapeutic results has been based on clinical criteria and on bacteriological criteria. If one accepts the evaluation of the Morphological Index (on smears or preferably on serial skin biopsies) all or not followed by inoculation into the mouse footpad, as a major criterium of therapeutic effect then the overall conclusion is that within 2 to 3 months of rifampicin at a dosage level of about $10 \mathrm{mg} / \mathrm{kg}$ in continuous daily administration or $15 \mathrm{mg} / \mathrm{kg}$ in intermittent administration, a bactericidal effect is achieved. Once again, however, we are limited by the available methodology.

In antibiotic therapy success and cure have of ten been attributed too exclusively to the rather simplistic assumption that a serum level in excess of the m.i.c. for the causative microorganism suffices.

The measurable and well documented decrease in viable bacilli in mouse footpads or even human skin lesions, when appropriate doses of rifampicin are administered seems to corroborate this idea.

We are all aware, however, that in the case of leprosy this is not a sufficient or final answer. It is already documented that relapses in patients under long standing DDS therapy have occurred and are most likely due to development originating from the so-called "dormant bacilli" located in muscle fibres and nerve sheaths. Little is known about the possible effect of rifampicin on these bacilli although some results have been claimed when rifampicin was used in association with other drugs.

Recent communications have drawn attention to the fact that DDS is acetylated and that as with INH (isoniazid) there may exist slow and rapid acetylators. So far no therapeutic relevance of this metabolic pattern is known. Rifampicin on the other hand is mainly de-acetylated to an active des-acetyl rifampicin, and if combined administration would be considered an investigation into combined pharmacokinetics might be indicated.

At least 2 additional types of information are lacking:

(a) We need to know more about rifampicin tissue penetration (e.g. in nerve and muscle fibres) and even more about rifampicin's intracellular and even probably intralysosomal penetration.

(b) We need to know more about "optimal" dosage, whether rifampicin is used alone or in combination.

For evident reasons related to the available evaluation criteria the main attention has till now been focused on active lepromatous leprosy.

The number of patients with borderline or tuberculoid forms of the disease is, however, far in excess of those with lepromatous leprosy, and it remains therefore a challenge to investigate the use of rifampicin in such cases provided suitable evaluation methods can be developed. In fact we are confronted with the paradoxical situation that rifampicin is a most, if not the most, active drug against Myco. leprae and in lepromatous leprosy, but we know neither whether rifampicin is or can be a curative drug nor how long it should be used. 
The third question is: how safe is rifampicin in the therapy of leprosy? In trying to provide an answer to this question we can first look at the considerable amount of data accumulated in the treatment of tuberculosis. Generally speaking one can say that rifampicin is a well tolerated and safe drug when administered continuously; there is no haematological, oto-vestibular or renal toxicity, and the data about hepatic side-effects do not exceed a few per cent of patients treated with the drug. Jaundice and or a deterioration in the hepatic function remain contra-indications for the use of rifampicin. The available data about leprosy do not differ substantially. Leprosy therapy is, however, confronted with some peculiar aspects related to the disease itself, one of them being reactive episodes. Different authors agree that under rifampicin administration the incidence of - reactions has been similar to that under DDS therapy and its intensity did not exclude continuation of therapy. Investigational use of highly successful intermittent rifampicin regimens in tuberculosis has been accompanied by several reports on side-effects of a different nature than those that were known under continuous therapy.

The intensity and incidence of these side-effects seem to be related to dosage and interval of doses. The most frequent of these side-effects is the so-called flu-syndrome with fever and arthralgia. Appearing within a few hours following rifampicin administration, it generally did not require interruption of therapy and could be managed in most cases by reduction in dosage or by a switch to continuous daily therapy.

The more serious side-effects such as thrombocytopenia, purpura, and anuria, which are fortunately rare, require immediate interruption of rifampicin therapy and forbid its further use.

Quite evidently these reports are a cause of justified concern; even more since similar accidents may occur under a rifampicin therapy that has been interrupted and restarted accidentally.

The clinical aspects of these side-effects have suggested that rifampicin induced immune complexes may be responsible.

In team work with a group of investigators of the University of Leuven, Dr Stevens and Dr Verbist, we are trying to investigate this hypothesis further. We know that rifampicin as such is not an antigen although it can probably conjugate with proteins and then elicit antibodies. Antigen-antibody reactions with mobilization of complement or activation of complement components are by analogy with other clinical syndromes considered to be involved in the different side reactions observed. Several teams of investigators have tried to monitor these immunological aspects in patients under rifampicin therapy in tuberculosis. One indirect method is based on complement fixation in a haemagglutination technique. The one which we use is based on immune complex precipitation (Ouchterlony).

The latter is certainly more specific but requires also the use of a stable rifamycin conjugate which is difficult to prepare.

Ongoing studies aim to look into the quantitative aspects and into the kinetics of this phenomenon. The precise relation between the clinical side-effects and the immune-complexes is not yet clearly established. We can only say that most likely it is not the mere presence of these "antibodies" (they might even be present spontaneously) that is responsible for the incidents, but most probably a critical ratio between antibodies and the hapten-carrier.

A lower incidence or practical absence of the side-effects just described has 
been observed in certain geographical areas (Zaire), and the hypothesis has been formulated that a different immunological reactivity could be involved.

These data, as mentioned, stem from tuberculosis research, and once more we are compelled to keep in mind the particular aspects of leprosy in its varied clinical manifestations. Little if any side-effects, and certainly no serious side-effects have, as far as I know, been reported in lepromatous patients under intermittent rifampicin therapy. The available information suggests in my opinion for the time being, 2 leads:

(1) A practical one: namely that any form of intermittent rifampicin therapy should be supervised with a close monitoring of the patients.

(2) An investigational one, which we are planning and which is aimed at looking into the immunological reactivity of different leprous patients before and during rifampicin therapy, with special regard to the presence or formation of these antibodies.

My fourth and last question is directly linked up with the previous ones. How practical is rifampicin in the therapy of human leprosy? The answer is evidently not only linked up with activity or efficacy and safety but also with socio-economic factors.

It has been ironically said that the major side-effect of rifampicin is its price. A large scale continuous daily use of rifampicin in the therapy of leprosy, mainly in developing countries, seems therefore wishful thinking.

The overall cost of therapy, however, is not just a matter of cost of chemotherapy but also of mobilizing the few available medical and paramedical personnel and facilities. Therefore any short therapy whether initial or definite that could reduce infectivity and substitute to any significant extent the life-long therapy regimens that are available now seems worth investigating.

The development of a short safe intermittent regimen could very well be a valid alternative even if it has to include an expensive drug.

Within short range I therefore dare suggest that further controlled trials be set up to investigate efficacy and safety of intermittent rifampicin or rifampicin containing regimens of different duration. Whenever possible these trials should be associated with immunological studies and optimally with a prolonged follow-up of patients.

Further ahead it is to be expected that new rifamycin derivatives with different pharmacokinetic properties will become available, first for animal investigation, and hopefully later for human use.

In summary it is my opinion that rifampicin has provided us with an extraordinarily active drug in lepromatous leprosy but that further research is mandatory to define its proper place and limitations in the overall therapy of leprosy. 